\title{
Renalase: A Novel Inflamatory Marker Post Living Donor Liver Transplantation
}

\author{
AHMED M. ABDUL GHANI, M.Sc.*; AHMED YAMANY, M.D.*; SAMAH ABD EL-HAMID, M.D.**; \\ MOSTAFA A. EL-SHAZLY, M.D.*** and MOHAMED Sh. MOGAWER, M.D.* \\ The Departments of Internal Medicine*, Clinical \& Chemical Pathology** and General Surgery \& Liver Transplantation***, \\ Faculty of Medicine, Cairo University
}

\begin{abstract}
Background: Transient elevation of aminotransferases, alkaline phosphatase and/or bilirubin are common findings post living donor liver transplantation, however, when enzymes showed marked or prolonged elevation, complications should be considered including acute rejection, hepatic artery thrombosis, infections or recurrence of the original disease.

Aim of the Study: To demonstrate whether serum renalase can be used as a potential biomarker of hepatocellular inflammation following living donor liver transplantation especially when combined with the standard LFT, CRP \& TLC.

Patients and Methods: The study was performed on 50 patients with End Stage Liver Disease (ESLD) undergone Living Donor Liver Transplantation (LDLT). Measurement of CBC, CRP, LFT and KFT were done daily for seven days and three months following liver transplantation. Immunoassay of serum renalase, six hours, one week and three months after transplantation.

Results: By comparing different laboratory data on day seven and third month to laboratory data on day one postliving donor liver transplantation, there was a statistically significant decline regarding AST, ALT, INR reflecting graft function, our study also revealed statistically significant positive correlation between serum Renalase levels and CRP and total leukocyte count three months after transplantation.

Conclusion: Serum renalase in the blood may help in early detection and possible prevention of hepatocellular inflammation and injury. In the future serum renalase may help in diagnosing acute cellular rejection post-living donor liver transplantation to improve the clinical outcomes of such major procedure.
\end{abstract}

Key Words: Renalase - Liver transplantation - Hepatocellular inflammation.

\section{Introduction}

PAUCITY of donor organs is a limiting factor in liver transplantation, while over 6000 liver trans-

Correspondence to: Dr. Ahmed M. Abdul Ghani,

The Department of Internal Medicine, Faculty of Medicine, Cairo University plant surgeries are performed annually in the United States, more than 2000 candidates die each year waiting liver transplantation. Living Donor Liver Transplantation (LDLT) provides a way to expand organs availability [1]

At present, any patient who has acute or chronic liver disease leading to inability to maintain normal quality of life, considered a candidate for liver transplantation. Most living donor liver transplant recipients experience a quality of life that it is much better than it was before transplantation, however they rarely achieve normal quality of life.

Following living donor liver transplantation surgery, nearly all patients experience abnormal liver function tests including aminotransferases, alkaline phosphatase and/or bilirubin, however, it is the time and magnitude of abnormal liver tests that determine the likely underlying pathology. Graft ischemia due to thrombosis of the hepatic artery is one of the most dread complications following transplantation because of the high degree of variability in clinical and laboratory presentation, so it is recommended to perform abdominal ultrasound with doppler assessment of hepatic and portal vessels especially intra-operative and early post-operative [2] .

Transaminases may be elevated post liver transplantation due to parenchymal liver disease including graft rejection, ischemia, recurrence of original disease, drug induced graft injury or primary infection including procedure related, donor related, recipient derived or hospital acquired [3] .

Human renalase protein consists of (FAD)binding region and an oxidase domain. At least four alternative splicing isoforms have been discovered in humans (renalase 1 to renalase 4 ). 
Sources include kidney, adipose tissue, liver and skeletal muscles. Renalase main functions are to degrade catecholamines like adrenaline (epinephrine) and noradrenaline (norepinephrine) in the blood circulation [4].

The purpose of this study is to measure renalase levels and markers of hepatocyte inflammation including AST, ALT and C-reactive protein after liver transplantation. In addition, we attempt to identify potential a clinical rule of renalase for early detection of early graft inflammation affecting liver transplants outcomes.

\section{Patients and Methods}

The present study was conducted on 50 patients with liver cirrhosis CHILD B-C undergone Living Donor Liver Transplantation (LDLT). Patients were recruited from El-Manial Specialized Hospital, Cairo University in the period from March 2014 to July 2017 and they were followed for three months. Their ages ranges from 20-64 years with a mean value of $49.28 \pm 9.28$. They were $36(72 \%)$ males and 14 (28\%) females. Patients agreed to participate in the study and informed consent was obtained.

\section{Inclusion criteria:}

1- Adult (more than 18 years old) patients with liver Cirrhosis CHILD B-C undergoing liver transplantation.

2- Both genders were included.

\section{Exclusion criteria:}

1- Evidence of AKI before surgery: Abrupt (within 48 hours) absolute increase in the serum creatinine concentration of $\geq 0.3 \mathrm{mg} / \mathrm{dL}$ from baseline.

2- Prior kidney transplantation and patients on potentially nephrotoxic drugs.

3- Chronic kidney disease patients; preoperative serum creatinine level greater than $4.0 \mathrm{mg} / \mathrm{dL}$, or end-stage renal disease (estimated glomerular filtration rate e-GFR $<60 \mathrm{~mL} / \mathrm{min}$ ).

4- Sepsis.

\section{Methods:}

Before transplantation all patients were subjected to full history and physical examination, including gender, age, comorbid diseases (diabetes, hypertension, and dyslipidemia), detailed drug history and Body Mass Index (BMI). Model for End-Stage Liver Disease (MELD) score was calculated for each recipient and abdominal Doppler ultrasonography was performed. Operative details were re- corded including ischemia time, intraoperative bleeding. After transplantation all patients were subjected to daily examinations as regards vital signs, drains and fluid balance, daily measurement of CBC, LFT and KFT was done for seven days as well as 3 months post liver transplantation. Levels of Calcineurin Inhibitor (CNI) were checked regularly. Doppler ultrasound was performed to assess graft homogeneity, biliary complications, portal trunk and hepatic vessels. Specific investigations including immunoassay of serum renalase six hours, one week and three months after transplantation.

\section{Renalase principle of the assay:}

Antibody specific to serum renalase coating the microtiter plate provided in this kit, samples were added to the appropriate microtiter plate wells with a biotin-conjugated polyclonal antibody preparation specific for serum Renalase. Each microplate well received avidin conjugated to Horseradish Peroxidase (HRP) and incubated, a substrate solution was added to each well. Only those wells that contain, biotin-conjugated antibody and enzyme-conjugated Avidin will exhibit a change in color. The reaction was terminated by the addition of a sulphuric acid solution and the color change was measured spectrophotometrically at a wavelength of $450 \mathrm{~nm} \pm 2 \mathrm{~nm}$. The concentration of the markers in the samples was then determined by comparing the O.D. of the samples to the standard curve.

\section{Detection range:}

Serum renalase level ranges from 0.78 to 50 $\mathrm{ng} / \mathrm{mL}$. The standard curve concentrations used for the ELISA's assay were ranged from zero to $50 \mathrm{ng} / \mathrm{ml}$.

\section{Calculation of results:}

Calculation the mean absorbance was done for each set of duplicate standards, controls and samples, and the average zero standard optical density was subtracted. Plotting the standard curve was performed using Sigma plot software, with standard concentration on the $\mathrm{x}$-axis and absorbance on the $\mathrm{y}$-axis. The best-fit straight line was drawn through the standard points.

\section{Statistical methods:}

All data were entered after coding using the statistical package SPSS Version 16 and summarized using mean, standard deviation for the quantitative variable. Comparisons between data was done using Analysis of Variance (ANOVA) with multiple comparisons post hoc test in quantitative 
variables. $p$-values less than 0.05 were considered as statistically significant. Correlations between measured parameters and laboratory data were done using spearman correlation coefficient.

\section{Declaration of ethics:}

The review board of Kasr Al-Aini Hospital approved to conduct this study. Oral and written informed consent was obtained from all patients according to Helsinki guidelines of research ethics.

\section{Results}

This study was performed prospectively on 50 patients had undergone living-donor liver transplantation. Their ages ranged from 20-64 years with a mean value of $49.28 \pm 9.28$. They were 36 (72\%) males and $14(28 \%)$ females.

Kidney, liver functions, complete blood counts and C-reactive protein data on day seven posttransplantation were recorded and compared to data collected on day one. There was a significant reduction of AST, ALT, INR and platelet counts with significant elevation of CRP, GGT and ALP at day seven post transplantation than at day 1 (Table 1).

Table (1): Mean and standard deviation of different laboratory data at day seven compared to day one.

\begin{tabular}{llll}
\hline The studied parameter & Day 1 & Day 7 & $p$-value \\
\hline CRP $(\mathrm{mg} / \mathrm{l})$ & $6.4 \pm 2$ & $14.3 \pm 2$ & 0.000 \\
Liver biochemical profile: & & & \\
Total bilirubin $(\mathrm{mg} / \mathrm{dl})$ & $5.3 \pm 2.4$ & $5.3 \pm 5$ & 0.94 \\
Direct bilirubin (mg/dl) & $2.8 \pm 1.1$ & $3.9 \pm 1.1$ & 0.20 \\
AST $(\mathrm{u} / \mathrm{l})$ & $375 \pm 230$ & $91 \pm 72$ & 0.001 \\
ALT (u/l) & $296 \pm 210$ & $143 \pm 85$ & 0.002 \\
Albumin (g/dl) & $3.2 \pm 1$ & $2.7 \pm 0.4$ & 0.13 \\
Total protein $(\mathrm{g} / \mathrm{dl})$ & $4.7 \pm 1.2$ & $4.4 \pm 2$ & 0.84 \\
INR & $2.2 \pm 0.7$ & $1.4 \pm 0.3$ & 0.000 \\
GGT (u/l) & $45.4 \pm 19$ & $118 \pm 60$ & 0.000 \\
ALP (u/l) & $49.5 \pm 16$ & $135 \pm 50$ & 0.000 \\
Complete blood count: & & & \\
Hemoglobin $(\mathrm{g} / \mathrm{dl})$ & $8.9 \pm 2.5$ & $9.2 \pm 1.2$ & 0.77 \\
Total leucocyte count & $8.7 \pm 2$ & $9.4 \pm 6$ & 0.80 \\
Platelets & $88.6 \pm 30$ & $76 \pm 20$ & 0.003 \\
\hline
\end{tabular}

Table (2): Showing different laboratory data at third month compared to day one by using paired $t$-test. There was a statistically significant decrease regarding AST, ALT, CRP, TLC, and statistically significant elevation of platelet count and hemoglobin.

Tables $(3,4)$ : Showing that renalase level was reduced after seven days and three months respectively in relation to day one post-operative with statistically significant difference by using paired $t$-test.

Table (2): Mean and standard deviation of different laboratory data at third month compared to day one.

\begin{tabular}{llll}
\hline The studied parameter & Day 1 & 3 months & $p$-value \\
\hline CRP (mg/l) & $6.4 \pm 2$ & $4.1 \pm 2$ & 0.002 \\
Complete blood count: & & & \\
Hemoglobin (g/dl) & $8.9 \pm 2.5$ & $12.3 \pm 1.5$ & 0.003 \\
Total leucocyte count & $8.7 \pm 2$ & $5.8 \pm 2$ & 0.004 \\
Platelets & $88.6 \pm 30$ & $162 \pm 56$ & 0.000 \\
Liver biochemical profile: & & & \\
Total bilirubin (mg/dl) & $5.3 \pm 2.4$ & $1.6 \pm 1.2$ & 0.000 \\
Direct bilirubin (mg/dl) & $2.8 \pm 1.1$ & $1 \pm 0.8$ & 0.000 \\
AST (u/l) & $375 \pm 230$ & $58.7 \pm 40$ & 0.000 \\
ALT (u/l) & $296 \pm 210$ & $55.7 \pm 30$ & 0.000 \\
Albumin (g/dl) & $3.2 \pm 1$ & $3.8 \pm 0.3$ & 0.11 \\
Total protein (g/dl) & $4.7 \pm 1.2$ & $6.2 \pm 0.9$ & 0.002 \\
INR & $2.2 \pm 0.7$ & $1.14 \pm 0.1$ & 0.02 \\
GGT (u/l) & $45.4 \pm 19$ & $57 \pm 43$ & 0.06 \\
ALP (u/l) & $49.5 \pm 16$ & $69 \pm 32$ & 0.07 \\
\hline
\end{tabular}

Table (3): Mean and SD of renalase at day 1 and 7.

\begin{tabular}{lccc}
\hline Variables & Day 1 & Day 7 & $p_{\text {-value }}$ \\
\hline Renalase $(\mathrm{ng} / \mathrm{ml})$ & $1.4 \pm 0.3$ & $1.25 \pm 0.4$ & 0.004 \\
\hline
\end{tabular}

Table (4): Mean and SD of renalase at day 1 and third month.

\begin{tabular}{lccc}
\hline Variables & Day 1 & 3 months & $p$-value \\
\hline Renalase $(\mathrm{ng} / \mathrm{ml})$ & $1.4 \pm 0.3$ & $1.03 \pm 0.4$ & 0.000 \\
\hline
\end{tabular}

Table (5): Showing statistically significant positive correlation between renalase versus AST at day seven by using Spearman correlation test.

Table (5): Correlation between renalase and different laboratory values at ay seven.

\begin{tabular}{lcc}
\hline \multirow{2}{*}{$\begin{array}{l}\text { The studied parameter } \\
\text { day seven }\end{array}$} & \multicolumn{2}{c}{ Renalase } \\
\cline { 2 - 3 } & Results (ng/ml) & $p$-value \\
\hline Liver biochemical profile: & & \\
$\quad$ Total bilirubin & 0.14 & 0.50 \\
Direct bilirubin & 0.12 & 0.44 \\
AST & 0.49 & 0.0001 \\
ALT & 0.22 & 0.12 \\
Albumin & -0.05 & 0.67 \\
Total protein & -0.08 & 0.70 \\
INR & 0.17 & 0.50 \\
GGT & 0.09 & 0.56 \\
ALP & 0.17 & 0.26 \\
Complete blood count: & & \\
Hemoglobin & 0.12 & 0.70 \\
Total leucocyte count & 0.20 & 0.16 \\
Platelets & 0.07 & 0.18 \\
CRP & 0.03 & 0.80 \\
\hline
\end{tabular}


Table (6): Showing statistically significant positive correlation between renalase versus CRP, TLC and non-significant negative correlation with serum albumin at the third month by using Spearman correlation test.

Table (6): Correlation between renalase and different laboratory values at third month

\begin{tabular}{lll}
\hline \multirow{2}{*}{$\begin{array}{l}\text { The studied parameter } \\
\text { third month }\end{array}$} & \multicolumn{2}{c}{ Renalase } \\
\cline { 2 - 3 } & Results $(\mathrm{ng} / \mathrm{ml})$ & $p$-value \\
\hline Liver biochemical profile: & & \\
Total bilirubin & 0.18 & 0.70 \\
Direct bilirubin & 0.10 & 0.64 \\
AST & 0.19 & 0.78 \\
ALT & 0.20 & 0.19 \\
Albumin & -0.04 & 0.69 \\
Total protein & -0.03 & 0.75 \\
INR & 0.47 & 0.0001 \\
GGT & 0.21 & 0.16 \\
ALP & 0.18 & 0.29 \\
Complete blood count: & & \\
Hemoglobin & 0.02 & 0.74 \\
Total leucocyte count & 0.48 & 0.001 \\
Platelets & 0.03 & 0.26 \\
CRP & 0.43 & 0.001 \\
\hline
\end{tabular}

\section{Discussion}

During the process of organ retrieval, storage and implantation, ischemic and inflammatory hepatocellular injury occur which is referred as Ischemia Reperfusion Injury (IRI), transaminases peak within 12 to 24 hours and normalize by 3-5 days after transplantation [5].

Abnormal liver tests post-liver transplantation are usually hepatocellular in pattern initially. Severity can range from mild rise in transaminase to a pattern of fulminant hepatic failure, with coagulopathy and encephalopathy, designated as Primary Nonfunction (PNF) [3].

Acute graft rejection 'usually severe enough to require treatment' that affects about one in four liver transplant recipients, most common mostly occurring within 90 post-transplantation, usually before the end of the first postoperative month, and neither the serum biochemical profile nor immunosuppression drug levels are useful in predicting the presence or severity of rejection. The diagnosis of rejection is histological, with biopsies typically obtained after a duplex ultrasound excluding hepatic artery thrombosis. Because causes other than rejection can present in an identical biochemical fashion, for example, viral hepatitis, empirical treatment of rejection is discouraged [6]
Renalase, a recently discovered FAD-binding protein, sources include adipose tissue, kidney, heart, liver, and skeletal muscles, effectively degrade catecholamines. Human Renalase mRNA include 4 variants [1-4], Renalase has 13.2\% amino acid identity of monoamine oxidase A (MAO-A), however it differs from MAO-A when it comes to catecholamine's degradation [7]

Sympathetic overactivity and generation of peroxidant molecules are crucial in liver fibrogenesis and cirrhosis. Impaired secretion of renalase into blood in patients with cirrhosis with chronic kidney disease may lead to organ damage and sympathetic overactivity [8].

In this study we tried to demonstrate the rule of serum renalase as a potential early inflammatory biomarker post-living donor liver transplantation with possible early detection of acute cellular rejection affecting liver transplant outcomes.

By comparing laboratory data from our postliver transplants patients day seven to laboratory data on day one, there was a statistically significant decline regarding AST, ALT, INR reflecting graft function. This is in agreement with Moon D. \& Lee S., demonstrating improved liver biochemistries steadily following liver transplantation surgery, as ischemia and reperfusion injury resolve [9].

Our study also showed that renalase level was reduced after seven days and three months in relation to day one post-operative with statistically significant difference.

We also found in our study statistically significant positive correlation between serum renalase levels serum aminotransferases at day seven, and at the third month significant positive correlation between serum renalase and CRP and total leukocyte count. This agree with the study done by Zhao B. et al., who demonstrated in their study that, Renalase exhibited an anti-inflammatory effect through suppressing renal-infiltrated macrophages [10].

To our knowledge, this might be the first study to demonstrate that there is statistically significant positive correlation between Renalase and aminotransferases, TLC and CRP post living donor liver transplantation. This might be related to early inflammatory process occurring with ischemia reperfusion injury post liver transplantation surgery and the anti-inflammatory effect of Renalase.

In the future, clinical treatment decisions based on the detection of renalase activities in the blood 
may help in early prediction of graft inflammation and possible early detection of cellular rejection to improve the clinical outcomes of liver transplantation.

\section{References}

1- NORTHUP P.G., ABECASSIS M.M., ENGLESBE M.J., et al.: Addition of adult-to-adult living donation to liver transplant programs improves survival but at an increased cost. Liver transplantation: Official publication of the American Association for the Study of Liver Diseases and the International Liver Transplantation Society, 15 (2): 148-62, 2009.

2- ABDELAZIZ O. and ATTIA H.: Doppler ultrasonography in living donor liver transplantation recipients: Intra-and post-operative vascular complications. World J. Gastroenterol., 21; 22 (27): 6145-72, 2016.

3- FEDORAVICIUS A.: Abnormal liver tests after liver transplantation. Michael Charlton clinical liver disease, 7 (4): 73-9, 2016.

4- DESIR G.V.: "Regulation of blood pressure and cardiovascular function by renalase". Kidney International, 76 (4): 366-70, 2009.
5- YU J., XIE S.F., XIA W.L., et al.: Risk factors of biliary complications after liver transplantation from donation after cardiac death. Journal of Zhejiang University. Medical Sciences, 43 (6): 664-9, 2014.

6- YAMABE H.: Banff schema for grading liver allograft rejection: An international consensus document. Hepatology, 25: 658-63, 1997.

7- LI X., HUANG R., XIE Z., et al.: Renalase, a new secretory enzyme: Its role in hypertensive-ischemic cardiovascular diseases. Medical science monitor: International Medical Journal of Experimental and Clinical Research, 20: 68892, 2014.

8- SANSOÉ G., ARAGNO M., MASTROCOLA R., AYOUBI M. and PAROLA M.: In experimental liver cirrhosis impaired secretion of renalase into blood may lead to organ damage and sympathetic overactivity. Digestive and Liver Disease, (49) 1: 20, 2017.

9- MOON D. and LEE S.: Liver Transplantation Gut. Liver, 3 (3): 145-65, 2009.

10- ZHAO B., ZHAO Q., LI J., et al.: Renalase Protects against Contrast-Induced Nephropathy in Sprague-Dawley Rats N. Ashton, ed. PLOS ONE, 10 (1): 0116583, 2015.

\section{دليل حيوى جديد لإلتهاب الخلايا الكبدية (Renalase)

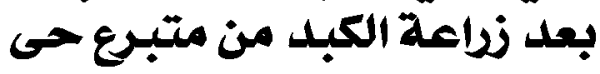

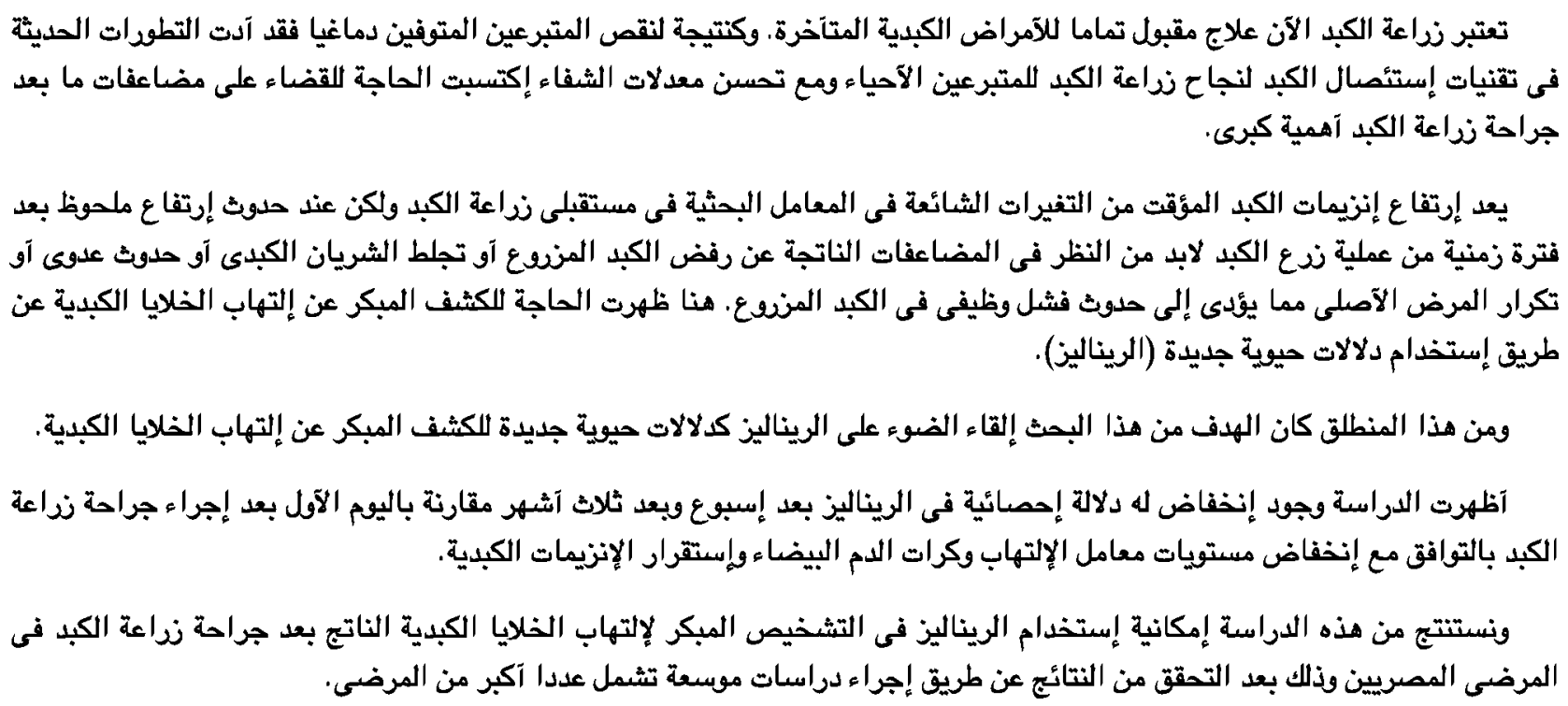

